\title{
Genome
}

\section{Fingerprint construction through genotyping by sequencing for applied breeding in Brassica rapa}

\begin{tabular}{|r|l|}
\hline Journal: & Genome \\
\hline Manuscript ID & gen-2021-0021.R1 \\
\hline Manuscript Type: & Article \\
\hline Author: & 15 -Sep-2021 \\
\hline Complete List of Authors: & $\begin{array}{l}\text { Li, Guoliang; Chinese Academy of Agricultural Sciences } \\
\text { Yue, Lixin; CASS IVF } \\
\text { Cai, Xu; CASS IVF } \\
\text { Li, Fei; CASS IVF } \\
\text { Zhang, Hui; CASS IVF } \\
\text { Zhang, Shujiang; CASS IVF } \\
\text { Zhang, Shifan; CASS IVF } \\
\text { Sun, Rifei; CASS IVF }\end{array}$ \\
\hline Keyword: & $\begin{array}{l}\text { Brassica rapa, genotyping by sequencing, finger-print, F1 lines, applied } \\
\text { breeding }\end{array}$ \\
\hline $\begin{array}{r}\text { Is the invited manuscript for } \\
\text { consideration in a Special } \\
\text { Issue? : }\end{array}$ & Not applicable (regular submission) \\
\hline & \\
\hline
\end{tabular}

\section{SCHOLARONE ${ }^{\text {M }}$ Manuscripts}




\section{Fingerprint construction through genotyping by sequencing for}

\section{applied breeding in Brassica rapa}

Guoliang Li ${ }^{1, \#}$, Lixin Yue ${ }^{1,2, \#, ~ X u ~ C a i ~}{ }^{1, \#}$, Fei Li ${ }^{1}$, Hui Zhang ${ }^{1}$, Shujiang Zhang ${ }^{1 *}$, Shifan Zhang ${ }^{1 *}$ \& Rifei Sun ${ }^{1 *}$

${ }^{1}$ Institute of Vegetables and Flowers, Chinese Academy of Agricultural Sciences, Zhongguancun, Nandajie No. 12, Haidian District, Beijing, 100081, China

${ }^{2}$ Shandong Key Laboratory of Greenhouse Vegetable Biology, Shandong Branch of National Vegetable Improvement Center, Huanghuai Region Vegetable Scientific Station of Ministry of Agriculture (Shandong), Institute of Vegetables, Shandong Academy of Agricultural Sciences, Jinan 250100, Shandong, China

\#These authors contributed equally to this work.

*For correspondence: zhangshujiang@caas.cn; zhangshifan@caas.cn; sunrifei@caas.cn

\section{Abstract}

This study evaluated genotyping by sequencing (GBS) protocol for fingerprinting Brassica rapa and the data derived were more reliable than the re-sequencing data of B. rapa. Of the 10 enzyme solutions used to analyze the numbers of genotypes and single nucleotide polymorphisms (SNPs) in B. rapa, five solutions showed better results, namely: A (HaeIII, 450-500 bp), E (RsaI+HaeIII, 500-550 bp), F (RsaI+HaeIII, 500-600 bp), G (RsaI+HaeIII, 'All' fragment), and $\mathrm{J}$ (RsaI+EcoRV-HF ${ }^{\circledR}, ' A l l '$ fragment). The five enzyme solutions showed less than $40 \%$ similarity in different individuals from various samples, and $90 \%$ similarity in between two individuals from one sample. The E enzyme solution was most suitable for fingerprinting $B$. rapa revealing well-distributed SNPs in the whole genome. Of the 82 highly inbred lines and $18 \mathrm{~F}_{1}$ lines of $B$. rapa sequenced by GBS in E enzyme solution, known parents of $10 \mathrm{~F}_{1}$ lines were verified and male parents were discovered for $8 \mathrm{~F}_{1}$ lines that had only known female parents. This study provided a valuable method for screening parents for F1 lines in B. rapa for applied breeding 
through efficient evaluation of GBS with varied library construction strategies.

Keywords: Brassica rapa; genotyping by sequencing; single nucleotide polymorphisms; fingerprinting; F1 lines; applied breeding

\section{Introduction}

In the past, reference genome sequences of many crops have provided a mass of information for understanding a crop's genomic variation and genome evolution. Owing to the genome-wide abundance of single-nucleotide polymorphisms (SNPs), their detection has become an efficient tool for molecular plant breeding. Based on the massive data generated from next-generation sequencing (NGS) technologies, genome studies have progressed rapidly, ranging from the assembly of draft genome sequences to genome diversity analysis. However, breeders require rapid and inexpensive SNP detection methods on various germplasm collections and breeding populations. Genotyping by sequencing (GBS) is one option that is highly efficient, relatively low cost, time-saving method for genome-wide SNP detection, because genome-wide reduced representation sequencing and utilizes NGS technology (Elshire et al., 2011; Lee et al., 2015).

The GBS protocol employs two different double-stranded adapters (barcode and common) that are ligated simultaneously to restriction fragments with "sticky" ends. This means that any adapters' combination (barcode/common, barcode/barcode, or common/common) may be paired with genomic DNA fragments (Elshire et al., 2011). The genome can be analyzed effectively by random fragments generated by different enzyme digestion combinations. GBS adopts the analysis of local location variation of the genome, and not the whole genome, which could save costs. In addition, GBS applications are becoming cost-effective with the decreasing cost of NGS and the increasing availability of reference genomes.

GBS facilitate the discovery of markers in huge numbers with their genotyping occurring simultaneously. For example, a bioinformatics pipeline and use-friendly tool for studying genomic diversity, TASSEL-GBS was designed for the efficient processing of raw GBS sequence data into SNP genotypes (Glaubitz et al., 2014). Further, the GBS technique has been utilized to construct a high-resolution genetic map and identify clubroot resistance $(C R)$ genes (Lee et al., 2015). In 
addition, GBS has been employed in an association study for the phenotype-genotype correlation analysis, and for genomic functional prediction in germplasm materials development for breeding in several crop species, such as maize (Elshire et al., 2011; Crossa et al., 2013; Romay et al., 2013), barley (Elshire et al., 2011; Poland et al., 2012), wheat (Poland et al., 2012), soybean (Sonah et al., 2013), and rice (Spindel et al., 2013).

GBS approaches have widely been applied to crops with well-sequenced reference genomes. The availability of reference genomes enable the discovery of sequence-based molecular markers and their association with agronomic traits for applied crop improvement (Hayward et al., 2012; Edwards et al., 2013; Zhang, 2019). Moreover, GBS has been successfully adapted for genetic mapping without reference genome sequences (Mascher et al., 2013).

Recently, genomic information has become increasingly available for Brassica species, such as B. rapa (Wang et al., 2011), B. oleracea (Liu et al., 2014; Parkin et al., 2014), and B. napus (Chalhoub et al., 2014). B. rapa is one of the three basic diploid Brassica species belonging to the evolutionary U's triangle (Nagaharu et al., 1935) and is an economically important vegetable crop consumed worldwide. However, the GBS technique has not been applied comprehensively yet in Brassica crops, which is one kind of approach for domestication studies in B. rapa (Yang et al., 2019). Hence, this study implemented GBS on two highly inbred B. rapa lines. GBS results were compared with the re-sequencing data to confirm the reliability of the GBS method. Of the 10 enzyme solutions tested for analyses of genotypes and SNPs, five enzyme solutions showed better results, namely: A (HaeIII, 450-500 bp), E (RsaI+HaeIII, $500-550$ bp), F (RsaI+HaeIII, 500600 bp), G (RsaI+HaeIII, 'All' fragment), and J (RsaI+EcoRV-HF ${ }^{\circledR}$, 'All' fragment). Notably, the SNPs from the E (RsaI+HaeIII, 500-550 bp) solution were well-distributed in the whole genome, therefore the E solution was the most suitable for sequencing analysis in B. rapa.

\section{Materials and methods}

\section{Plant materials and DNA extraction}

Two B. rapa highly inbred lines, namely the Chinese cabbage 'Kefeng80' (S1) and the Pakchoi cabbage 'Xiangxiu' (S2) were used in this study. S1 and S2 each had five individual samples 
designated as follows: S1-1, S1-2, S1-3, S1-4, S1-5, S2-1, S2-2, S2-3, S2-4, and S2-5. All 82 B, rapa highly inbred lines and $18 \mathrm{~B}$. rapa $\mathrm{F}_{1}$ lines were planted in a greenhouse. Genomic DNAs from 10 plants were extracted from 2-g samples of young leaves, following the modified cetyltrime thylammonium bromide (CTAB) protocol (Allen et al., 2006). The quality and quantity of the DNA were measured using Nanodrop 2000 (Thermo Fisher Scientific Inc., USA).

\section{Enzyme solutions}

Ten enzyme solutions (A to J, Table 1) used in this study were comprised of a single enzyme or a combination of two of three enzymes: HaeIII, RsaI, and $E c o R V-\mathrm{HF}^{\circledR}$ and then paired with the different fragment sizes: $400-450$ bp, $450-500$ bp, $400-500$ bp, $500-550$ bp, $500-580$ bp, $500-600 \mathrm{bp}$, or All at 400-600 bp.

Table 1. Composition of the 10 enzyme solutions used in this study

\begin{tabular}{|c|c|c|c|c|}
\hline Enzyme solution & Enzyme name & Fragment size (bp) & Tag numbers* & Enzyme efficiency ${ }^{* *}$ \\
\hline A & HaeIII & $450-500$ & 16,370 & $91.61 \%$ \\
\hline B & HaeIII & All & 300,000 & $93.31 \%$ \\
\hline $\mathrm{C}$ & RsaI & $500-580$ & 57,494 & $92.21 \%$ \\
\hline $\mathrm{D}$ & RsaI & All & 300,000 & $81.81 \%$ \\
\hline E & RsaI+HaeIII & $500-550$ & 57,827 & $86.27 \%$ \\
\hline $\mathrm{F}$ & RsaI+HaeIII & $500-600$ & 105,614 & $79.49 \%$ \\
\hline G & RsaI + HaeIII & All & 300,000 & $86.83 \%$ \\
\hline $\mathrm{H}$ & $R s a \mathrm{I}+E c o R \mathrm{~V}-\mathrm{HF}^{\circledast}$ & $400-450$ & 55,968 & $93.70 \%$ \\
\hline I & $R s a \mathrm{I}+E c o R \mathrm{~V}-\mathrm{HF}^{\circledR}$ & $400-500$ & 105,731 & $93.40 \%$ \\
\hline $\mathrm{J}$ & $R s a \mathrm{I}+E c o R \mathrm{~V}-\mathrm{HF}^{\circledR}$ & All & 300,000 & $91.66 \%$ \\
\hline
\end{tabular}

"Predicted tags based on the Brassica rapa genome enzyme sites

${ }^{* *}$ Through multiple tests, the efficiencies of an enzyme or enzymes digesting the target sites in a certain time.

\section{Library sequencing}

Genome DNA was digested to completion with HaeIII, RsaI, and EcoRV-HF ${ }^{\circledR}$ (NEB). A 
single-nucleotide 'A' overhang was added to the digested fragments with Klenow Fragment (3e di) (NEB) and deoxyadenosine triphosphate (dATP) at $37^{\circ} \mathrm{C}$. Then, the Duplex Tag-labeled Sequencing adapters (PAGE purified, Life Technologies) were ligated to the A-tailed DNA with $\mathrm{T}_{4}$ DNA ligase. The PCR reaction was performed using diluted restriction-ligation samples, deoxynucleoside triphosphate (dNTP), Q5 ${ }^{\circledR}$ High-Fidelity DNA Polymerase and PCR primers: AATGATACGGCGACCACCGA and CAAGCAGAAGACGGCATACG (PAGE purified, Life Technologies). The PCR productions were purified using Agencourt AMPure XP beads (Beckman Coulter, High Wycombe, UK) and pooled. The pooled sample was separated by electrophoresis in a $2 \%$ agarose gel. Fragments with $400-450$ bp, $450-500$ bp, $400-500$ bp, 500-550 bp, $500-580$ bp, 500-600 bp, or All (with indexes and adaptors) in size were excised, purified using QIAquick Gel Extraction Kit (QIAGEN). The gel-purified product was sequenced on the Illumina HiSeq 2500 system (Illumina, Inc; San Diego, CA, U.S.) following the manufacturer's recommendations.

\section{Raw sequenced data processing and SNP genotyping calling}

Based on the predicted genomic information for each enzyme combination, the raw data of each sample had a depth of more than 10×, and the NGSQCToolkit_v2.3.3 (Patel \& Jain, 2012) was used to filter low quality reads. To extract reliable variant information, Burrows-Wheeler Alignment tool (BWA) (Li \& Durbin, 2009) was used to map all clean data of each sample on $B$. rapa genome (Wang et al., 2011) and SamTools (Li et al., 2009) was used to extract the whole genome variations. Moreover, a complete analysis was conducted using a method of controlling the amount of clean data. For example, $150 \mathrm{Mb}$ of clean reads were selected randomly, and the same method of aligning and extracting SNP was used to obtain the final SNP of each sample.

\section{Analyses of the similarity among the individuals}

All detected SNP variations were analyzed for the similarity of the two varieties. First, the genotype loci of the two $B$. rapa varieties were compared with $B$. rapa reference sequences. Then, the genotyping results of the same locus were extracted.

Second, sites were counted that had SNP in at least one or two samples. Figure 1 shows the III and IV types of SNPs removed, and only retained the types I and II SNPs. The similarities of SNPs 
were computed between two different individuals from one sample, or different samples using the formula below.

Similarity $=[($ the number of II type SNPs $) /($ the number of $(\mathrm{I}+\mathrm{II})$ types of SNPs $)] * 100 \%$.

\section{Results}

\section{High reliability of genotyping by sequencing}

The A (HaeIII, 450-500 bp) enzyme solution by GBS was selected and its results were compared with the results of the re-sequence method for reliability. Comparison results showed that SNPs extracted by GBS was highly reliable. The S1-1, S1-2, S1-3, S1-4, and S1-5 materials were re-sequenced and more than $10 \times$ clean data was generated that was used to compare with the GBS data. Approximately $300 \mathrm{Mb}$ data were chosen from the GBS and re-sequencing methods in calling SNPs. From GBS method, the average genotype number of the S1-1, S1-2, S1-3, S1-4, and S1-5 individuals was 318,795 , while the average number in re-sequencing method was $2,305,192$ from the reS1-1, reS1-2, reS1-3, reS1-4, and reS1-5 individuals. The genotype numbers extracted from GBS were lower than the values in the re-sequencing method maybe due to GBS only considered some fragments to sequence, while the re-sequencing method chose the whole genomic sequences. The average numbers of the same genotypes between GBS and re-sequencing methods were 304,685 , which accounted for $96 \%$ of the average numbers of the genotypes from GBS. In addition, the shared genotype locus $(304,603)$ between GBS and re-sequencing methods accounted for $99.97 \%$ of the numbers of same genotypes (Table 2), thereby suggesting that the GBS method is a reliable way to analyze the differences among individuals.

Table 2. Comparative analysis between GBS and re-sequencing.

\begin{tabular}{ccccc}
\hline re-sequence & GBS & Shared genotyped locus & No. of Same Genotypes & Reliability \\
\hline reS1-1 & S1-1 & 331,865 & 331,947 & $99.98 \%$ \\
reS1-2 & S1-2 & 319,843 & 319,930 & $99.97 \%$ \\
reS1-3 & S1-3 & 265,949 & 266,010 & $99.97 \%$ \\
reS1-4 & S1-4 & 315,239 & 315,335 & $99.97 \%$ \\
reS1-5 & S1-5 & 290,121 & 290,203 & $99.97 \%$
\end{tabular}




$\begin{array}{lll}\text { Average } \quad 304,603 & 304,685 & 99.97 \%\end{array}$

Note: reS1-1: re-sequencing sample 1-1; S1-1: GBS sample 1-1.

In this study, to reduce uncertain genotyping due to low-coverage sequences, SNPs were filtered and only considered reads with $>10 \times$ coverage as well as removed those with high-depth reads, over than $100 \times$ coverage to alleviate duplicate genome or repeat sequences. Notably, in the same data size, GBS showed far less numbers of genotypes than those from re-sequencing.

Many PCR reduplications were found after analyzing the GBS data. Hence, the ratio of the invalid data in re-sequencing and GBS was analyzed, and results showed 50\% and 5\% invalid data ratio in GBS and in re-sequencing, respectively (Table 3). The shared genotype numbers were less, but the SNP numbers from E (RsaI+HaeIII, $500-550 \mathrm{bp})$ enzyme solution was sufficient for further analyses. Additionally, the SNPs from GBS were well-distributed in the whole genome for fingerprinting in B. rapa. The features of GBS method were relatively straightforward, robust, and cost-effective, so GBS protocol is currently being applied in numerous species.

Table 3. The ratio of invalid data in the re-sequence and GBS methods

\begin{tabular}{rllr}
\hline Methods & $<3 \times$ reads & $<50 \times$ reads & $\begin{array}{c}\text { Invalid } \\
\text { Ratio }\end{array}$ \\
\hline re-sequence & 119,863 & $2,473,390$ & $4.85 \%$ \\
GBS-AS1-1 & $20,570,995$ & $46,635,528$ & $44.11 \%$ \\
GBS-BS1-1 & $3,914,334$ & $7,205,838$ & $54.32 \%$ \\
GBS-CS1-2 & $9,899,913$ & $15,591,117$ & $63.50 \%$ \\
GBS-DS1-2 & $7,093,596$ & $16,485,892$ & $43.03 \%$
\end{tabular}

Note: To confirm the ratio of the invalid data, four GBS sequencing samples were chosen (GBS-AS1-1: GBS, A enzyme solution, sample1-1) and then results were compared with the re-sequence data.

\section{Analyses of genotypes and SNPs numbers through genotyping by sequencing}

The data sizes from the 10 enzyme solutions by the GBS method were different largely, thus the same data size $(150 \mathrm{M})$ was extracted randomly from each individual. After filtering the raw reads, 
the clean reads were aligned to the reference genome, and calling the genotypes and SNPs was performed. The numbers of genotypes from B (HaeIII, 'All' fragment) and C (RsaI, 500-580 bp) enzyme solutions were less than the genotypes in other enzyme solutions (Fig. 2). Interestingly, the numbers of genotypes in S1 were higher than those in S2 (Fig. 2). The use of enzyme solutions A (HaeIII, $450-500$ bp), E (RsaI+HaeIII, $500-550$ bp), F (RsaI+HaeIII, $500-600$ bp), G (RsaI+HaeIII, 'All' fragment), and $\mathrm{J}\left(R s a \mathrm{I}+E c o R \mathrm{~V}-\mathrm{HF}^{\circledR}\right.$, 'All' fragment) resulted in more genotypes than in other enzyme solutions. It is worth noting that the G (RsaI+HaeIII, 'All' fragment) and $\mathrm{J}\left(R s a \mathrm{I}+E c o R V-H F^{\circledR}\right.$, 'All' fragment) enzyme solutions selected all the fragment size (Table 1) and the A (HaeIII, 450-500 bp) enzyme solution used only one enzyme, which may have fewer enzyme sites than two enzymes, whereas the E, and F (RsaI+HaeIII, 500-600 bp) enzyme solutions used two enzymes (RsaI+HaeIII) and chose 500-550 bp and 500-600 bp fragments, respectively (Table 1).

The numbers of SNPs from B and C enzyme solutions were less than in the other enzyme solutions (Fig. 3), which was consistent with the genotypes. Unlike the genotype, the numbers of SNPs in S1 were lower than those in S2 (Fig. 4). Based on the analysis of SNPs, the A, D, E, F, G, I, and J enzyme solutions were preferable. Furthermore, D, G, and J enzyme solutions were paired with all the fragment sizes (Table 1) and the A (HaeIII, 450 - 500 bp) enzyme solution used only one enzyme. Meanwhile, the E, F, and I enzyme solutions used two enzymes (Table 1). Comprehensive analysis of genotypes and SNPs showed that E (RsaI+HaeIII, $500-550$ bp) had SNPs better-distributed in the whole genome (Fig. 4) compared to F (RsaI+HaeIII, 500 - 600 bp), although both solutions used similar combination of two enzymes. Moreover, the E enzyme solution generated sufficient amounts of SNPs for fingerprint analysis of B. rapa. The number of genotypes and SNPs from the E enzyme solution was the highest, thereby suggesting the suitability of GBS method for B. rapa fingerprinting.

\section{Analysis of the SNPs distribution and the similarities between individuals from one or different samples}

Analysis of the density of S1-1 under E (RsaI+HaeIII, 500-550 bp) enzyme solution showed 
SNPs that were distributed throughout the 10 chromosomes that may be well-distributed in the genome (Fig. 4). The number of SNPs was 2,805 in A09 that was in most abundance in the 10 chromosomes. The numbers of SNPs were 1,331 in A04 and 1,424 in A10, which were nearly half of those in A09. The number of SNPs in A09 was similar with the sum of those in A03 and in A06. The numbers of SNPs were about 2,000 in all A01, A02, A05, A07, and A08 chromosomes (Fig. 4). Overall, the SNPs extracted from the GBS method covered the whole genome, and were well-distributed in all 10 chromosomes, implying the great reliability of SNPs from GBS method for fingerprint identification of the germplasm resources in B. rapa. In addition, analyses of the similarity of different plants of the same samples or between different samples revealed less than $40 \%$ similarity among different samples, and above $90 \%$ similarity among different plants in the same samples (Fig. 5) that showed distinctiveness of B. rapa collection from different sources.

\section{Screening the male parent for hybridization $\left(F_{1}\right)$ in applied breeding}

Using the $\mathrm{E}(R s a \mathrm{I}+H a e I I I, 500-550 \mathrm{bp})$ enzyme solution, 82 highly inbred lines and $18 \mathrm{~F}_{1}$ lines of Brassica rapa were sequenced (Table 4). In addition, GBS method verified and confirmed the known two parents of $10 F_{1}$ lines. Meanwhile, the other eight $F_{1}$ lines only have known female parents, and through GBS the male parents were identified (Table 4). Based on the B. rapa reference genome 3.0, the variants of 100 lines were extracted and the neighbor-joining (NJ) tree was constructed using all the variants (Fig. 6). Of the $10 \mathrm{~F}_{1}$ lines, five lines: 42, 50, 51, 52, and 54 were good matches of their parents. Based on the NJ tree, the male parents of eight F1 lines (non-known parents) were established (Table 4).

Table 4. Identification of male and female parents of $18 \mathrm{~F}_{1}$ lines of Brassica rapa through genotype by sequencing

\begin{tabular}{ccccc}
\hline Serial Numbers & $\mathrm{F}_{1}$ lines & $\begin{array}{c}\text { Female } \\
\text { parent }\end{array}$ & Male parent & $\begin{array}{c}\text { Concluded } \\
\text { parent }\end{array}$ \\
\hline 1 & 42 & 28 & 31 & - \\
2 & 46 & 16 & 15 & - \\
3 & 47 & 41 & 17 & - \\
4 & 48 & 13 & 56 & - \\
5 & 49 & 23 & 14 & -
\end{tabular}




\begin{tabular}{ccccc}
6 & 50 & 25 & 29 & - \\
7 & 51 & 26 & 30 & - \\
8 & 52 & 32 & 40 & - \\
9 & 54 & 37 & 36 & - \\
10 & 55 & 34 & 33 & - \\
11 & 43 & 24 & $?$ & 21 \\
12 & 44 & 20 & $?$ & 25 \\
13 & 45 & 18 & $?$ & 70 \\
14 & 53 & 35 & $?$ & 36 \\
15 & 56 & 22 & $?$ & 48 \\
16 & 57 & 30 & $?$ & 51 \\
17 & 58 & 19 & $?$ & 20 \\
18 & 59 & 39 & $?$ & 38 \\
\hline
\end{tabular}

232

233

\section{Discussion}

With the development of NGS, may raw sequence data can be obtained (Metzker 2010; Shendure \& Aiden, 2012; Edwards et al., 2013; Kilpinen et al., 2013) that simple and convenient bioinformatics analysis methods can fully develop (Van et al., 2007, 2008). An NGS-based method, GBS uses high-throughput genotyping of large numbers of SNP markers from several populations. Because of its relative low cost and high-throughput capacity, the GBS method has already been applied in many studies of various plant species (Poland et al., 2012; Chen et al., 2013). The growth of next-generation DNA sequencing technologies has led to a rapid increase in sequence-based genotyping for applications including diversity assessment, genome structure validation and gene-trait association. For example, a research study has established a skim-based GBS method for crop plants and has applied this approach to genotype-segregating populations of B. napus and Cicer arietinum (Bayer et al., 2015). Additionally, GBS technology was used to densely genotype a DH broccoli population segregating for head quality under high-temperature field conditions for three summer seasons that may help accelerate breeding for heat tolerance in broccoli (Branham et al., 2017). GBS also resulted in numbers of polymorphisms from near-isogenic lines (NILs) and some SNPs were identified in three various wax synthesis genes (Branham \& Farnham, 2017) that could be helpful in genome editing to improve B. rapa qualities (Chen et al., 2020). 
In additions, GBS has been used in almost all the cruciferous crops, employed to construct a high-resolution genetic map, used to identify clubroot resistance (CR) gene and identified 43,821 SNPs in B. oleracea (Lee et al., 2015). Likewise, a study has shown the genotypic characterization of 79 B. napus genotypes by calculating genetic distance based on sequence-related amplified polymorphism (SRAP) and GBS in association with a NJ clustering algorithm (Lees et al., 2016). Another study showed that GBS of the parental lines and $\mathrm{BC} 1$ plants resulted in about $1.32 \mathrm{M}$ sequences from T19 were aligned into the reference genome of $B$. rapa with 0.4-fold coverage, and 1.77 M sequences with 0.5-fold coverage (Yu et al., 2017). GBS also was applied to construct a high-density SNP-based genetic linkage group with 1,465 bin markers, covering 1,285.77 cM of genome length, with an average genetic distance of $0.88 \mathrm{cM}$ between adjacent bin markers in $B$. oleracea (Li et al., 2019). QTL associated with three wild-type related traits including relative root length (RRL), relative hypocotyl length (RHL) and relative fresh weight (RFW) were dissected in B. napus by GBS, and identified 1,468 and 1,450 binned SNPs (Ding et al., 2020). Based on SNP identified from GBS, an association study of flowering time as well as crude fat and fatty acid contents was conducted in 46 rapeseed mutant lines derived from gamma rays (Ryu et al., 2021). Further, 623,026,394 clean data reads were generated with 6.6 million reads on average, demonstrating that SNPs are efficient tools for mutant screening and provided a basis for improving the rapeseed's oil qualities (Ryu et al., 2021). With the completion of cruciferous crop genome sequencing, the B. rapa (Wang et al., 2011), B. oleracea (Liu et al., 2014; Parkin et al., 2014), and B. napus (Chalhoub et al., 2014), GBS has been more widely used in cruciferous crops for gene mapping, high-resolution genetic map construction, genetic variation mining, and association study for agricultural traits, among other research applications.

\section{Ethics approval and consent to participate}

Not Applicable

\section{Consent for publication}

Not Applicable 


\section{Availability of data and materials}

Not Applicable

\section{Authors' contributions}

RS designed the study. GL, LY, and XC collected and analyzed the data and drafted the manuscript. SZ, SZ, FL, and HZ helped in data collection. GL and RS approved final version of manuscript.

\section{Acknowledgments}

This work was performed at the Key Laboratory of Biology and Genetic Improvement of Horticultural Crops, Ministry of Agriculture, Beijing, China. We thank LetPub (www.letpub.com) for its linguistic assistance during the preparation of this manuscript.

\section{Funding}

This work was founded by Beijing Natural Science Foundation (6212030), the National Natural Science Foundation of China (32102373), and the National Natural Science Foundation of China (31772302).

\section{Competing interests}

The authors declare that they have no competing interests.

\section{References}

1. Allen, G.C.; Flores-Vergara, M.A.; Krasynanski, S.; Kumar, S.; \& Thompson, W.F. A modified protocol for rapid DNA isolation from plant tissues using cetyltrimethyl ammonium bromide. Nature protocols. 2006, 1: 2320-2325.

2. Bayer, P.E.; Ruperao, P.; Mason, A.S.; Stiller, J.; Chan, C.K.K.; Hayashi, S.; \& Varshney, R.K. High-resolution skim genotyping by sequencing reveals the distribution of crossovers and gene conversions in Cicer arietinum and Brassica napus. Theoretical and Applied 
Genetics. 2015, 128: 1039-1047.

3. Branham, S.E.; \& Farnham, M.W. Genotyping-by-sequencing of waxy and glossy nearisogenic broccoli lines. Euphytica. 2017, 213: 84.

4. Branham, S.E.; Stansell, Z.J.; Couillard, D.M.; \& Farnham, M.W. Quantitative trait loci mapping of heat tolerance in broccoli (Brassica oleracea var. italica) using genotyping-bysequencing. Theoretical and Applied Genetics. 2017, 130: 529-538.

5. Chalhoub, B.; Denoeud, F.; Liu, S., Parkin, I.A.; Tang, H.; Wang, X.; \& Corréa, M. Early allopolyploid evolution in the post-Neolithic Brassica napus oilseed genome. Science. 2014, 345: 950-953.

6. Chen, Q.; Ma, Y.; Yang, Y.; Chen, Z.; Liao, R.; Xie, X.; \& Yang, C. Genotyping by genome reducing and sequencing for outbred animals. PLoS One. 2013, 8: e67500.

7. Chen, Y.T.; Mao, W.W.; Liu, T.; Feng, Q.Q.; Li, L.; \& Li. B.B. Genome editing as a versatile tool to improve horticultural crop qualities. Horticultural Plant Journal. 2020, 6 : $372-384$.

8. Cheng, F.; Sun, R.; Hou, X.; Zheng, H.; Zhang, F.; Zhang, Y,; et al. Subgenome parallel selection drives morphotype diversification and convergent crop domestication in Brassica rapa and brassica oleracea. Nature genetics. 2016, 48: 1218-1224.

9. Crossa, J.; Beyene, Y.; \& Kassa, S. Genomic prediction in maize breeding populations with genotyping-by-sequencing. Genes Genomes Genetics. 2013, 3: 1903-1926.

10. Ding, X.; Xu, J.; Huang, H.; Xing, Q.; Shen, M.; Cheng, Y.; \& Zhang, X. Unraveling waterlogging tolerance-related traits with QTL analysis in reciprocal intervarietal introgression lines using genotyping by sequencing in rapeseed (Brassica napus L.). Journal of Integrative Agriculture. 2020, 19, 1974-1983.

11. Edwards, D.; Batley, J.; \& Snowdon, R.J. Accessing complex crop genomes with nextgeneration sequencing. Theoretical and Applied Genetics. 2013, 126: 1-11.

12. Elshire, R.J.; Glaubitz, J.C.; Sun, Q.; Poland, J.A.; Kawamoto, K.; Buckler, E.S.; \& Mitchell, S.E. A robust, simple genotyping-by-sequencing (GBS) approach for high diversity species. PloS One. 2011, 6: e19379.

13. Glaubitz, J.C.; Casstevens, T.M.; Lu, F.; Harriman, J.; Elshire, R.J.; Sun, Q.; \& Buckler, E.S. 
TASSEL-GBS: a high capacity genotyping by sequencing analysis pipeline. PloS One. 2014, 9: e90346.

14. Hayward, A.; Vighnesh, G.; Delay, C.; Samian, M.R.; Manoli, S.; Stiller, J.; \& Batley, J. Second-generation sequencing for gene discovery in the Brassicaceae. Plant Biotechnology Journal. 2012, 10: 750-759.

15. Kilpinen, H.; \& Barrett, J.C. How next-generation sequencing is transforming complex disease genetics. Trends in Genetics. 2013, 29: 23-30.

16. Lee, J.; Izzah, N.K.; Choi, B.S.; Joh, H.J.; Lee, S.C.; Perumal, S.; Nou, I.S. Genotyping-bysequencing map permits identification of clubroot resistance QTLs and revision of the reference genome assembly in cabbage (Brassica oleracea L.). DNA Research. 2015, 23: 2941.

17. Lees, C.; Li, G.; \& Duncan, R. Characterization of Brassica napus L. genotypes utilizing sequence-related amplified polymorphism and genotyping by sequencing in association with cluster analysis. Mol Breeding. 2016, 36, 155.

18. Li, H.; \& Durbin, R. Fast and accurate short read alignment with Burrows-Wheeler transform. Bioinformatics. 2009, 25: 1754-1760.

19. Li, H.; Handsaker, B.; Wysoker, A.; Fennell, T.; Ruan, J.; \& Homer, N. The sequence alignment/map format and Samtools. Bioinformatics. 2009, 25: 2078-2079.

20. Li, X.; Kong, C.; Yu, H.; Liu, X.; Fang, Z.; Liu, Y.; \& Zhang, Y. Identification of a major QTL for seed number per silique in cabbage (Brassica oleracea L. var. capitata) using genotyping by sequencing. Euphytica. 2019, 215, 1-13.

21. Liu, S.; Liu, Y.; Yang, X.; Tong, C.; Edwards, D.; Parkin, I.A.; et al. The Brassica oleracea genome reveals the asymmetrical evolution of polyploid genomes. Nature communications. 2014, 5: 3930 .

22. Mascher, M.; Wu, S.; Amand, P.S.; Stein, N.; \& Poland, J. Application of genotyping-bysequencing on semiconductor sequencing platforms: a comparison of genetic and referencebased marker ordering in barley. PLoS One. 2013, 8: e76925.

23. Metzker, M.L. Sequencing technologies - the next generation. Nature reviews genetics. 2010, 11: 31-46. 
24. Nagaharu, U. Genome analysis in brassica with special reference to the experimental formation of B. napus and peculiar mode of fertilization. Japanese Journal of Botany. 1935, $389-452$.

25. Parkin, I.A.; Koh, C.; Tang, H.; Robinson, S.J.; Kagale, S.; Clarke, W.E.; \& Denoeud, F. Transcriptome and methylome profiling reveals relics of genome dominance in the mesopolyploid Brassica oleracea. Genome Biology. 2014, 15: R77.

26. Patel, R.K.; \& Jain, M. NGS QC Toolkit: a toolkit for quality control of next generation sequencing data. PloS One. 2012, 7: e30619.

27. Poland, J.A.; \& Rife, T.W. Genotyping-by-sequencing for plant breeding and genetics. The Plant Genome. 2012, 5: 92-102.

28. Romay, M.C.; Millard, M.J.; \& Glaubitz, J.C. Comprehensive genotyping of the USA national maize inbred seed bank. Genome Biology. 2013, 14: R55.

29. Ryu, J.; Lyu, J; Kim, D.; Koo, K.; Yang, B.; Jo, Y.; Kim, S.; Kwon, S.; Ha, B.; Kang, S.; Kim, J.; Ahn, J. Single Nucleotide Polymorphism (SNP) Discovery and Association Study of Flowering Times, Crude Fat and Fatty Acid Composition in Rapeseed (Brassica napus L.) Mutant Lines Using Genotyping-by-Sequencing (GBS). Agronomy. 2021, 11, 508.

30. Shendure, J.; \& Aiden, E.L. The expanding scope of DNA sequencing. Nature Biotechnology. 2012, 30: 1084-1094.

31. Sonah, H.; Bastien, M.; Iquira, E.; Tardivel, A.; Légaré, G.; Boyle, B.; \& Belzile, F. An improved genotyping by sequencing (GBS) approach offering increased versatility and efficiency of SNP discovery and genotyping. PloS One. 2013, 8: e54603.

32. Spindel, J.; Wright, M.; Chen, C.; Cobb, J.; Gage, J.; Harrington, S.; \& McCouch, S. Bridging the genotyping gap: using genotyping by sequencing (GBS) to add high-density SNP markers and new value to traditional bi-parental mapping and breeding populations. Theoretical and Applied Genetics. 2013, 126: 2699-2716.

33. Van, N.J.; Hogers, R.C.; Janssen, A.; Yalcin, F.; Snoeijers, S.; Verstege, E.; \& Van, M.J. Complexity reduction of polymorphic sequences (CRoPS ${ }^{\mathrm{TM}}$ ): a novel approach for largescale polymorphism discovery in complex genomes. PloS One. 2007, 2: e1172.

34. Van Tassell, C.P.; Smith, T.P.; Matukumalli, L.K.; Taylor, J.F.; Schnabel, R.D.; Lawley, 
C.T.; \& Sonstegard, T.S. SNP discovery and allele frequency estimation by deep sequencing of reduced representation libraries. Nature Methods. 2008, 5: 247-252.

35. Wang, X.; Wang, H.; Wang, J.; Sun, R.; Wu, J.; Liu, S.; et al. The genome of the mesopolyploid crop species Brassica rapa. Nature genetics. 2011, 43: 1035-1039.

36. Yang, Z.M.; Li, G.X.; Tieman, D., \& Zhu, G.T. Genomics approaches to domestication studies of horticultural crops. Horticultural Plant Journal. 2019, 5: 240-246.

37. Yu, F.; Zhang, X.; Peng, G.; Falk, K.; Strelkov, S.; \& Gossen, B. Genotyping-by-sequencing reveals three QTL for clubroot resistance to six pathotypes of Plasmodiophora brassicae in Brassica rapa. Scientific reports. 2017, 7, 1-11.

Zhang, L.S. Advance of horticultural plant genomes. Horticultural Plant Journal. 2019, 5: 229230.

\section{Figures}

Fig. 1. Schematic diagram of the similarity between two individuals.

Fig. 2. The average numbers of genotypes in two Brassica rapa samples: Chinese cabbage 'Kefeng80' (S1) and Pakchoi cabbage 'Xiangxiu' (S2) analyzed in 10 enzyme solutions

Fig. 3. The average numbers of SNPs found in two Brassica rapa samples: Chinese cabbage 'Kefeng80' (S1) and Pakchoi cabbage 'Xiangxiu' (S2) in 10 enzyme solutions

Fig. 4. The SNPs distributions of the S1-1 dealt by E (RsaI+HaeIII, 500-550 bp) enzyme solution in $\mathrm{A} 01$ to $\mathrm{A} 10$.

Fig. 5. The similarity analysis among different individuals from one or different samples. (S1-1/S1-2: the similarity between S1-1 and S1-2 individuals)

Fig. 6. The neighbor-joining tree of the 82 highly inbred lines and $18 \mathrm{~F}_{1}$ lines of Brassica rapa. (The colored label identifies the 10 known $\mathrm{F}_{1}$ and their corresponding parents.) 


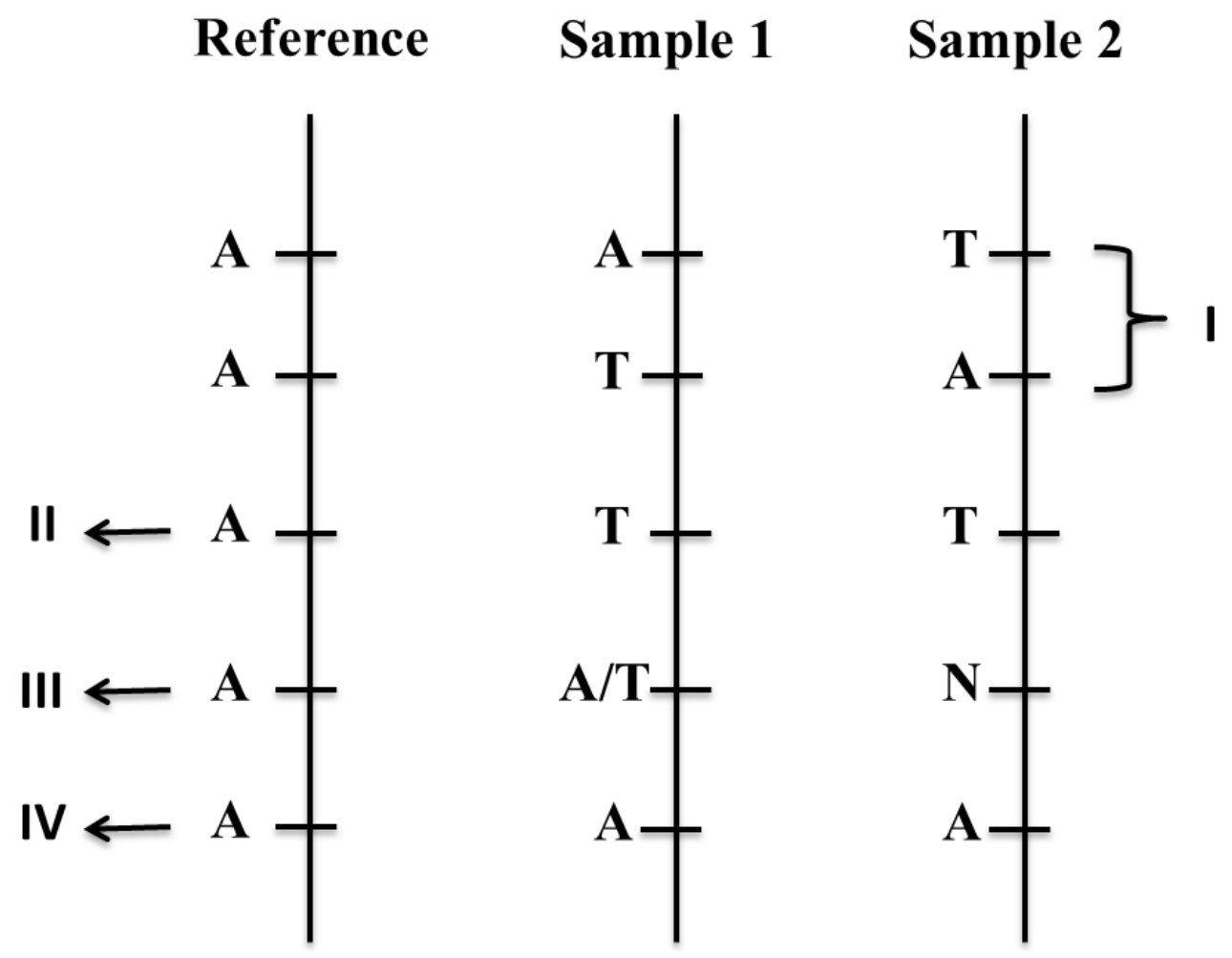

Fig. 1. Schematic diagram of the similarity between two individuals.

$238 \times 180 \mathrm{~mm}(96 \times 96 \mathrm{DPI})$ 


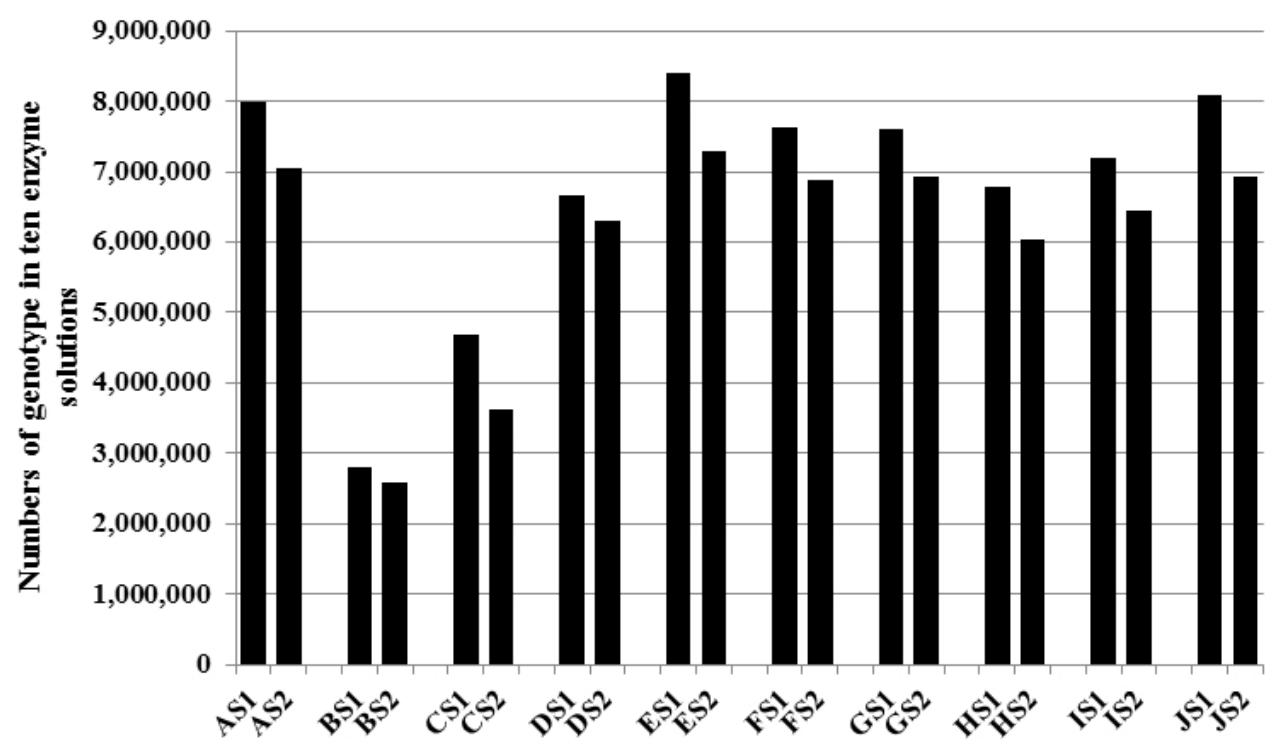

Fig. 2. The average numbers of genotypes in two Brassica rapa samples: Chinese cabbage 'Kefeng80' (S1) and Pakchoi cabbage 'Xiangxiu' (S2) analyzed in 10 enzyme solutions 


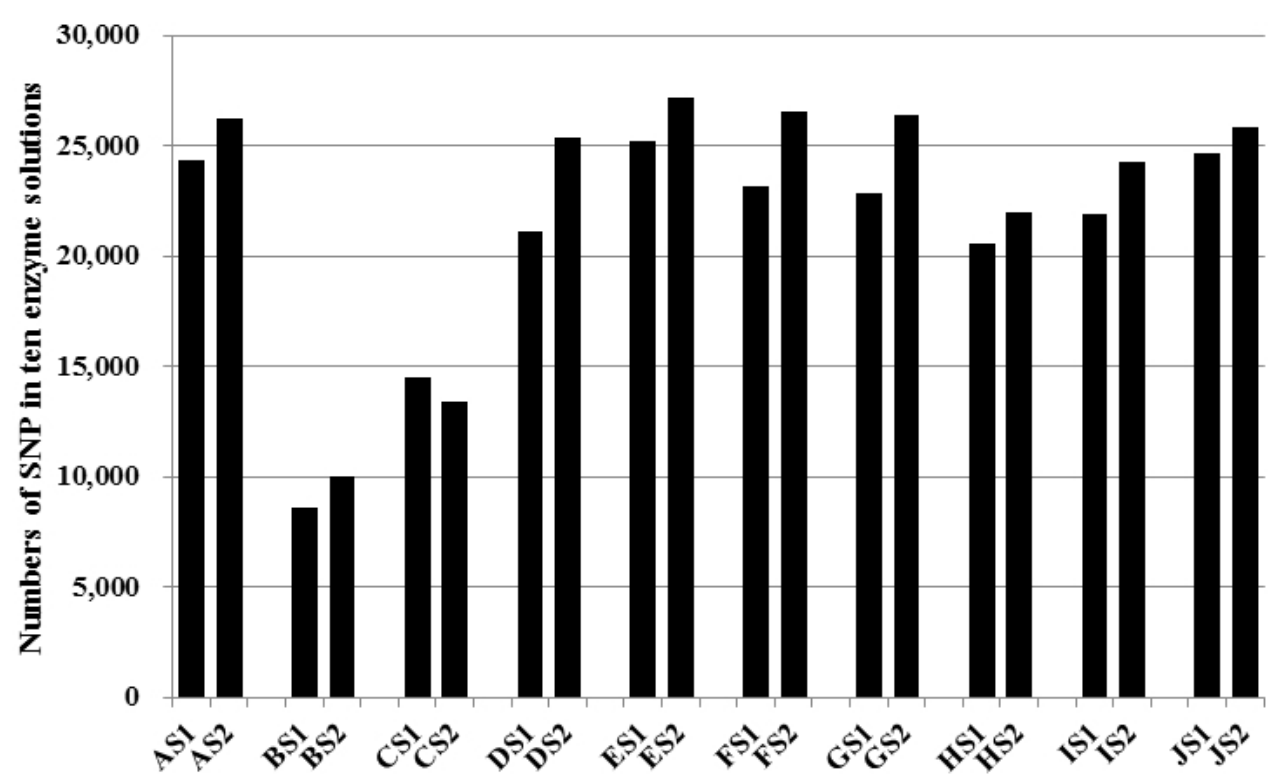

Fig. 3. The average numbers of SNPs found in two Brassica rapa samples: Chinese cabbage 'Kefeng80' (S1) and Pakchoi cabbage 'Xiangxiu' (S2) in 10 enzyme solutions 


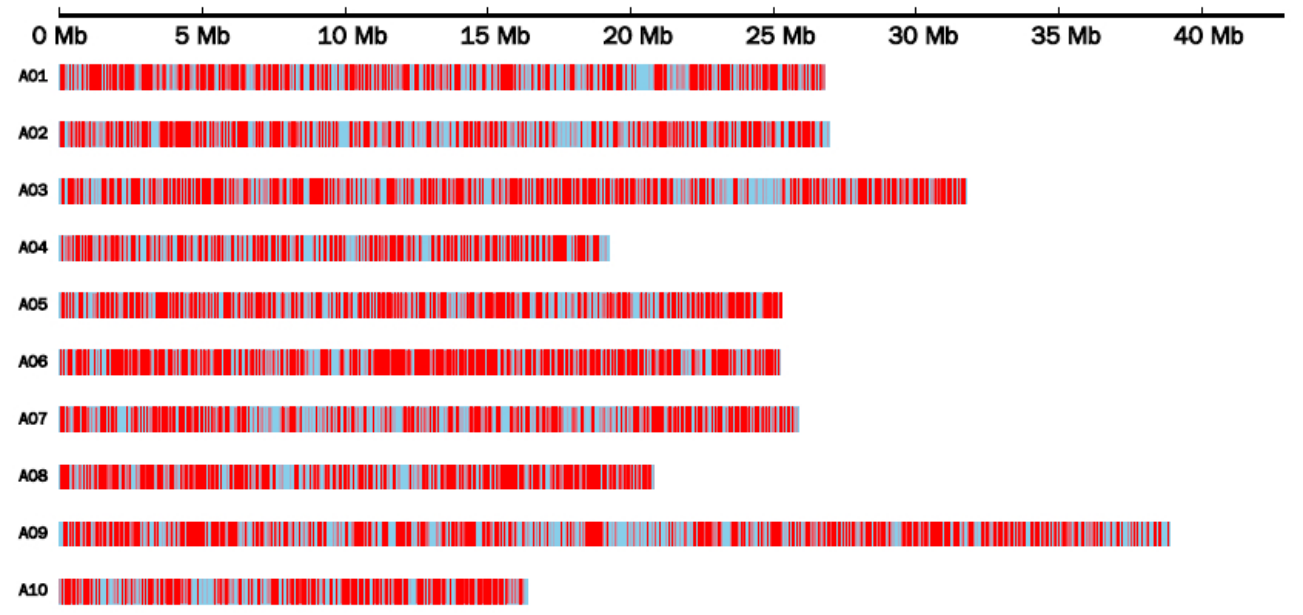

Fig. 4. The SNPs distributions of the S1-1 dealt by E (RsaI+HaeIII, 500-550 bp) enzyme solution in A01 to A10. 


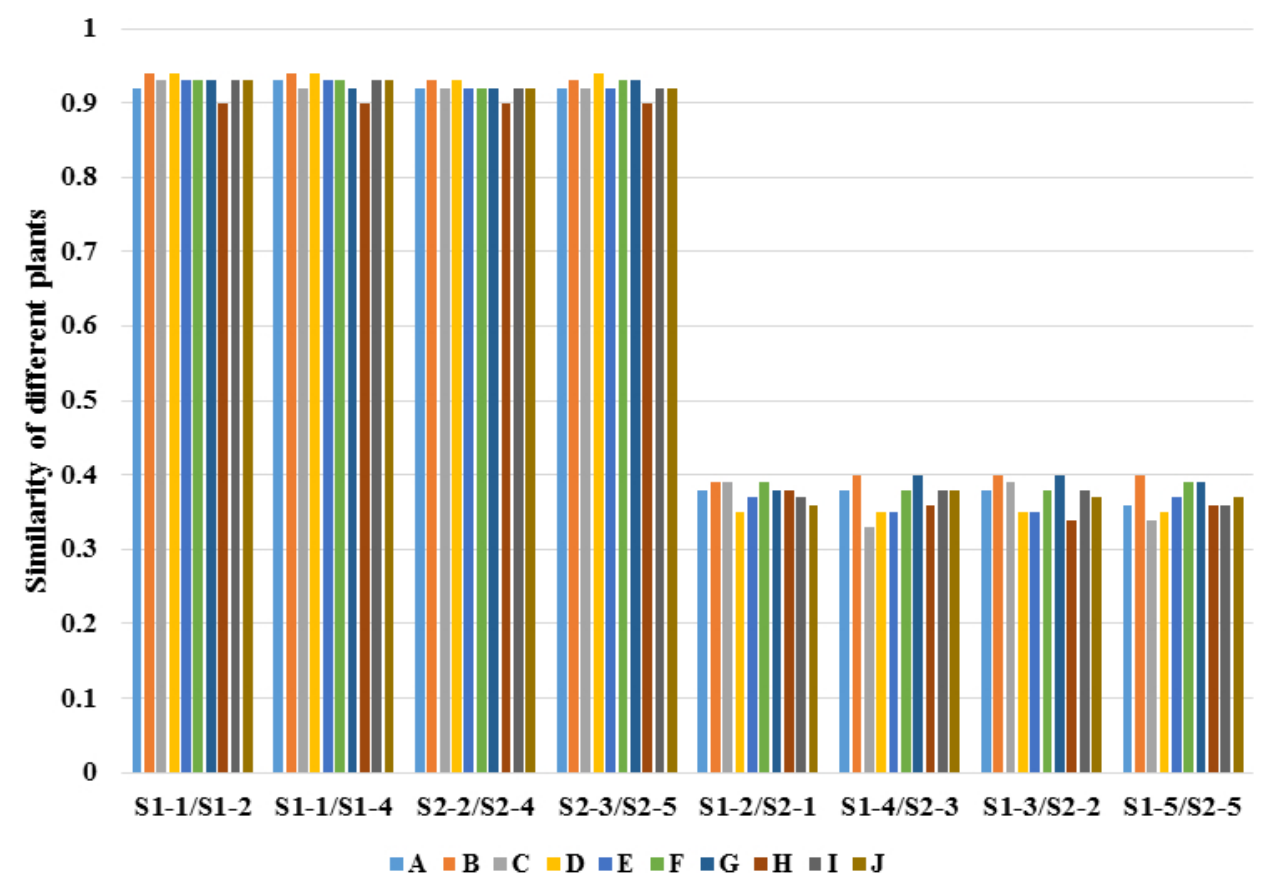

Fig. 5. The similarity analysis among different individuals from one or different samples. (S1-1/S1-2: the similarity between S1-1 and S1-2 individuals) 


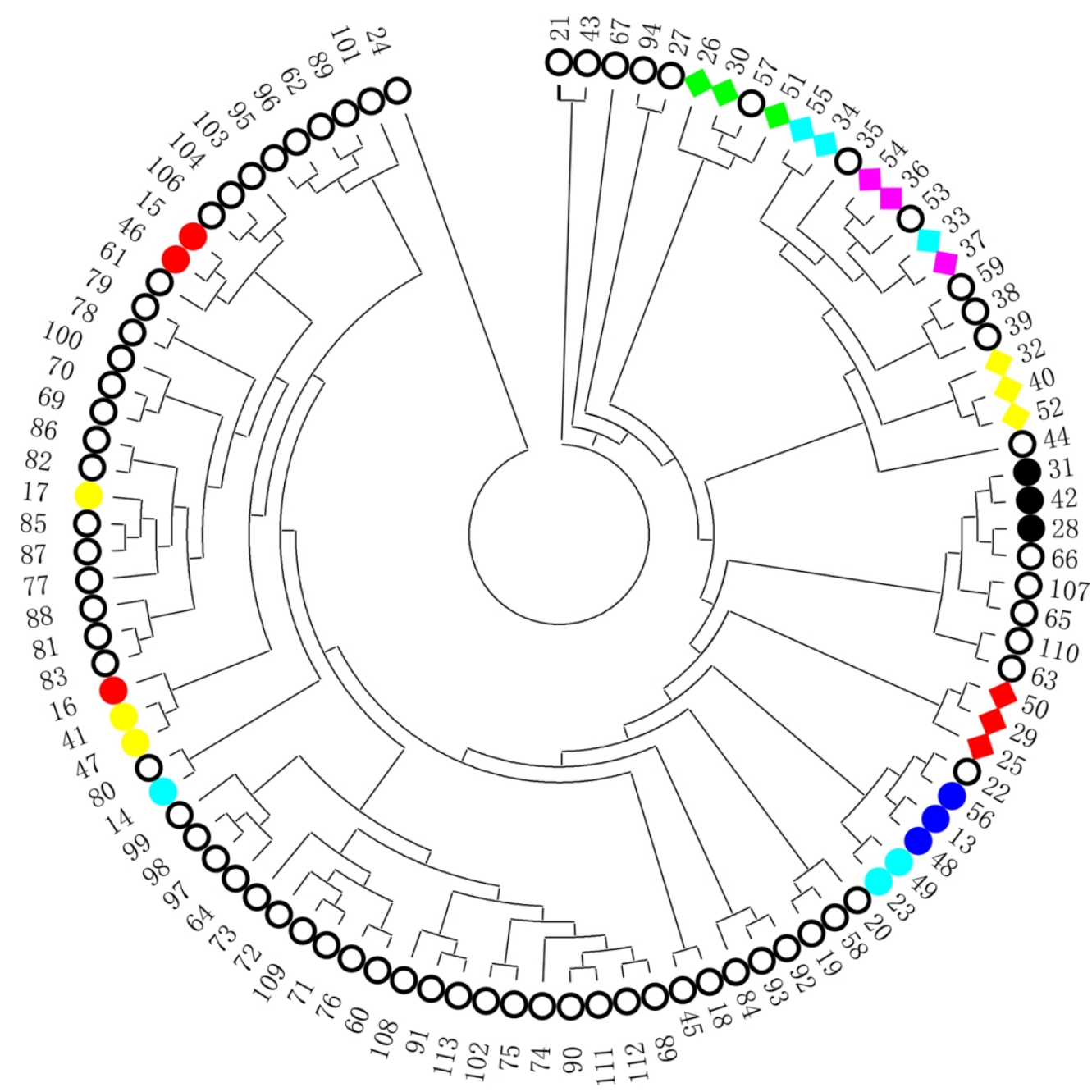

Fig. 6. The neighbor-joining tree of the 82 highly inbred lines and 18 F1 lines of Brassica rapa. (The colored label identifies the 10 known $\mathrm{F} 1$ and their corresponding parents.)

$200 \times 200 \mathrm{~mm}(200 \times 200$ DPI $)$ 